PharmacoEconomics \& Outcomes News 879, p12 - 29 May 2021

\title{
Expediting COVID-19 vaccination increases QALYs and saves costs in the USA
}

A modelling study has shown that expediting COVID-19 vaccination reduces hospitalisations and deaths, increases quality-adjusted life years (QALYs) and is cost saving in the USA.

The researchers adapted a computational model that represented the US population ( $\mathrm{n}=327$ million), its interactions, COVID-19 spread and vaccination, as well as potential COVID-related economic outcomes over 3 years. The vaccine was assumed to prevent infection with no waning immunity. Experimental scenarios compared various vaccine coverage levels (from $10 \%-90 \%$ ) and vaccination rates, with varying times to achieve the various coverage levels (180-360 days). Third-party payer cost data included total direct medical costs (including vaccination and hospitalisation), and the societal perspective included direct and indirect costs (productivity loss from COVID-19 illness). Costs were reported in 2020 values and were discounted at 3\% per annum.

When a given vaccination coverage is achieved in 270 days with $70 \%$ vaccine efficacy, each $1 \%$ in coverage would avert 876800 cases, varying according to the number of people already vaccinated: each $1 \%$ increase from $40 \%$ to $50 \%$ coverage would prevent 1.5 million COVID-19 cases, 56240 hospitalisations, 6660 deaths, gain 77590 qualityadjusted life years (QALYs), save \$US602.8 million in direct medical costs and \$1.3 billion in indirect costs. Speeding up the time to vaccination coverage to 180 days could save another 5.8 million cases, 215,790 hospitalisations, 26370 deaths, 206520 QALYs, $\$ 3.5$ billion in direct medical costs, and $\$ 4.3$ billion in indirect costs.

The authors point out that "increasing vaccination coverage can be as important as or even more important than having a higher efficacy vaccine. . . Our results also show how the time it takes to reach different coverage levels can affect the impact and value of the COVID-19 vaccine, showing the importance of achieving high coverage levels as soon as possible". 